\title{
A model for universal time scale of vortex ring formation
}

\author{
Kamran Mohseni \\ Division of Engineering and Applied Science, 104-44, California Institute of Technology, Pasadena, \\ California 91125 \\ Morteza Gharib \\ Graduate Aeronautical Laboratory, California Institute of Technology, Pasadena, California 91125
}

(Received 23 April 1998; accepted 16 June 1998)

\begin{abstract}
An analytical model for predicting the universal time scale for formation of vortex rings generated through impulsively started jets is considered. The model is based on two assumptions, namely the validity of the slug model in simulating the discharge process of the fluid out of the cylinder and the approximation of the vortex at the pinch off moment by a vortex in the Norbury family. The nondimensional stroke length $L / D$ (referred to as "formation number," following Gharib et al. [J. Fluid Mech. 360, 121 (1998)]) predicted by the model satisfactorily matches the experimental observation of Gharib et al. The model introduces two nondimensional parameters that govern the limiting formation number: nondimensional energy $E_{\text {nd }}$ and circulation $\Gamma_{\text {nd }}$. The predicted value of $E_{\text {nd }}$ matches very well with the experimental data. It is also predicted that there is a limiting value for the nondimensional circulation in the range $1.77 \lesssim \Gamma_{\mathrm{nd}} \lesssim 2.07$. (C) 1998 American Institute of Physics. [S1070-6631(98)00810-1]
\end{abstract}

There is strong evidence in support of the notion that the formation of vortex rings is mainly an inviscid process. This can be easily checked by comparing the relaxation time toward a steady vortex ring with the viscous diffusion time at high Reynolds numbers. The long-time behavior of an axisymmetric Euler equation is characterized by an infinite number of invariants. ${ }^{1}$ Any accurate model for vortex ring formation must respect these invariants.

In a recent paper, Gharib et al. ${ }^{2}$ have demonstrated that a time scale with a narrow range of values characterizes the formation of axisymmetric vortex rings. This time scale is the time that the vortex ring pinches off from its generating axisymmetric jet. Here we refer to the nondimensional stroke length $L / D$ as the "formation number." The relation between this formation number and the corresponding time scale is given by $L / D=U_{p} t / D$, where $U_{p}$ is the average piston velocity. Gharib et al. ${ }^{2}$ demonstrated that this observed time, beyond which larger rings are not possible, is a direct manifestation of the variational principle proposed by Kelvin and Benjamin for steady axis-touching rings. For predicting the actual value of the formation number, they used a nondimensional energy parameter obtained from the experimental results. The purpose of this paper is to show that the formation number can be predicted analytically, based on simple assumptions on the formation process.

To develop our model we make two assumptions. First that the discharge process of the fluid out of the cylinder can be predicted by a slug model. Second, that at the pinch-off moment the invariants of the resulting vortex ring can be approximated by those of a vortex in the family of Norbury vortices. ${ }^{3}$ While real physical vortex rings have peakier vorticity distributions $(\omega)$ than those of the uniform vorticity density $(\omega / r)$ of the Norbury family of vortices, the quantitative behavior, in particular the stream lines, is very similar. ${ }^{4}$ These considerations along with the fact that Norbury family of vortices are very well documented suggest that for the limiting process under consideration the Norbury vortices can be considered as a first approximation to the more general case of vorticity distributions. Peakiness of the vorticity profiles in this context is considered in more detail in Ref. 2. Furthermore the experimental results provided by Gharib et al. ${ }^{2}$ for different velocity profiles of the piston and exit conditions suggest that the limiting formation number is not very sensitive to the vorticity distributions. This is physically manifested in constant values for nondimensional energy $E_{\text {nd }}$ and circulation $\Gamma_{\text {nd }}$ (both quantities are defined later) for the leading vortex of a pinched off jet.

The Norbury family of vortices are steady solutions to the axisymmetric Euler equations. They are characterized by the parameter $\alpha$ with $0<\alpha \leqslant \sqrt{2}$. Here $\alpha$ is defined as the nondimensional mean core radius. The family ranges from vortex rings of small cross section, where $\alpha \rightarrow 0$, to Hill's spherical vortex, which has the parameter value $\alpha=\sqrt{2}$. The vorticity density $\omega / r$ in each ring is a constant. It has already been shown by $\mathrm{Wan}^{5}$ that the Norbury family of vortices are the solution of a maximization problem on the energy function subjected to fixed impulse and circulation.

Before developing the model we would like to make an interesting point. Since the kinetic energy per unit density, circulation, and impulse per unit density for a slug of fluid with diameter $D$, length $L$, and with uniform velocity $U_{p}$ are given by

$$
\begin{aligned}
& E=\frac{1}{8} \pi D^{2} L U_{p}^{2}=\left(U_{p} / 2\right) I, \\
& \Gamma=\frac{1}{2} L U_{p}, \\
& I=\frac{1}{4} \pi D^{2} L U_{p}=\frac{1}{2} \pi D^{2} \Gamma,
\end{aligned}
$$

we can write 


$$
E=I \Gamma / L .
$$

Hence for fixed impulse and circulation [and therefore from (3) fixed $R$ ] the minimum stroke length $L$, which results in a Norbury vortex, maximizes the energy.

The corresponding invariant quantities for the Norbury vortices are nondimensionalized as follows:

$$
\begin{aligned}
& E=(\Omega \alpha l)^{2} l^{3} E_{N}, \\
& \Gamma=(\Omega \alpha l) l \Gamma_{N}, \\
& I=(\Omega \alpha l) l^{3} I_{N},
\end{aligned}
$$

where the subscript $N$ refers to the corresponding nondimensional quantity, for the Norbury vortices, $\Omega=\omega / r$ is the vorticity density, and $l$ is the vortex radius (see Ref. 3). For future reference we note that the dimensional translation velocity $U_{\text {tr }}$ and the nondimensional translation velocity $U_{N}$ of the Norbury vortices are related by

$$
U_{\mathrm{tr}}=(\Omega \alpha l) U_{N} \text {. }
$$

An estimate for $U_{\text {tr }}$ may be obtained from the following relation $^{6}$

$$
\left.(\partial E / \partial I)\right|_{\text {fixed } \Gamma \text { and vol. }}=U_{\text {tr }},
$$

which for the slug model $[(1)-(3)]$ results in

$$
U_{\mathrm{tr}}=U_{p} / 2 \text {. }
$$

Note that similar results have been reported by other researchers using different approaches. Therefore Eq. (8) can be transformed using (10) to

$$
U_{p}=2(\Omega \alpha l) U_{N} \text {. }
$$

Our approach here is to calculate the corresponding parameter $\alpha$ of the resulting Norbury vortex and get the nondimensional stroke length $L / D$ which corresponds to it. In doing so we adopt an approach which is independent of the way that the nondimensionalizations are done. The final stage is governed by the invariants of the motion for the slug model. These are the kinetic energy, the total circulation, and the impulse. Equating these quantities for the slug model $[(1)-(3)]$ with the corresponding quantities in the Norbury vortices $[(5)-(7)]$ and using (11) we obtain

$$
\begin{aligned}
& \frac{L}{D}=\sqrt{\pi / 2} I_{N}^{1 / 2} \Gamma_{N}^{3 / 2} / E_{N}, \\
& \frac{L}{D}=\sqrt{\pi / 2} \Gamma_{N}^{3 / 2} / U_{N} I_{N}^{1 / 2}, \\
& E_{N}=U_{N} I_{N} .
\end{aligned}
$$

According to Norbury ${ }^{3}$ the right-hand side of Eqs. (12) and (13) and Eq. (14) are only a function of $\alpha$. The nondimensional stroke length $L / D$ from Eqs. (12) and (13), as a function of $\alpha$, are shown in Fig. 1. Their intersection yields the parameter $\alpha$ of the resulting Norbury vortex. The corresponding stroke length $L / D$ for the resulting Norbury vortex is approximately equal to 3 . Finally note that the $\alpha$ of the resulting Norbury vortex can also be calculated from the last equations among (12)-(14), which is the same as what is predicted in Fig. 1. This consistency is in fact a manifesta-

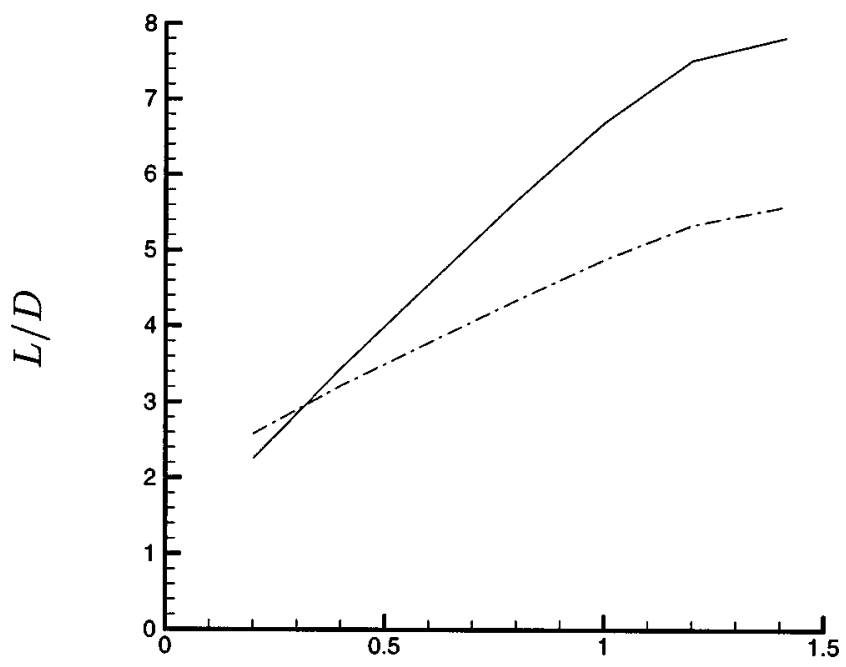

$\alpha$

FIG. 1. Relation between the formation number $(L / D)$ and the parameter of Norbury's family of vortices $(\alpha)$. - Eq. (12); ---:Eq. (13).

tion of the existing relation [Eq. (9)] between the invariants of motion for axisymmetric flows which for the slug model is represented by (11).

Equations (12) and (13) may also be derived using dimensional analysis. This will also show that these are the only independent relations involved in the prediction of the pinch-off process. We assume that the variables involved in the pinch-off process are $L / D, U_{p}$ [or equivalently $U_{\mathrm{tr}}$, which is obviously related to $U_{p}$ by (9) and (10), $L / D$ $\left.=2 t U_{\mathrm{tr}} / D\right]$, and the invariants of motion $T, \Gamma, I$. Therefore we can write

$$
L / D=f\left(E, \Gamma, I, U_{p}\right) .
$$

A straightforward dimensional analysis reveals that the limiting $L / D$ only depends on the nondimensional energy $E_{\text {nd }}$ and circulation $\Gamma_{\text {nd }}$ defined as follows:

$$
\begin{aligned}
& E_{\mathrm{nd}}=E / \Gamma^{3 / 2} I^{1 / 2}, \\
& \Gamma_{\mathrm{nd}}=\Gamma / I^{1 / 3} U_{p}^{2 / 3} .
\end{aligned}
$$

The nondimensional energy $E_{\text {nd }}$ has already been defined in Ref. 7 and used in Ref. 2. Equating the nondimensional energy and circulation between the slug model and the Norbury vortices and using (1)-(3), (5)-(7), (10), and (11) result in Eqs. (12) and (13). Therefore there are only two independent curves in Fig. 1, where their intersection gives the formation number associated with the pinch-off process.

Note that in practice the translation velocity of real vortex rings are more than $U_{p} / 2$ (which is what the slug model predicts). If we repeat the calculations leading to Eq. (13), with $U_{\text {tr }}$ varying between $0.5 U_{p}$ and $0.6 U_{p}$ we obtain 3.0 $\lesssim L / D \leqq 4.5$ (see Fig. 2). This range of stroke length for the pinch-off process is quite consistent with the observed values in experiments by Gharib et al. ${ }^{2}$

A few remarks are in order. The fact that the Norbury parameter $\alpha$ corresponding to the pinch-off process is much smaller than $\sqrt{2}$ for the Hill's vortex is an indication of the 


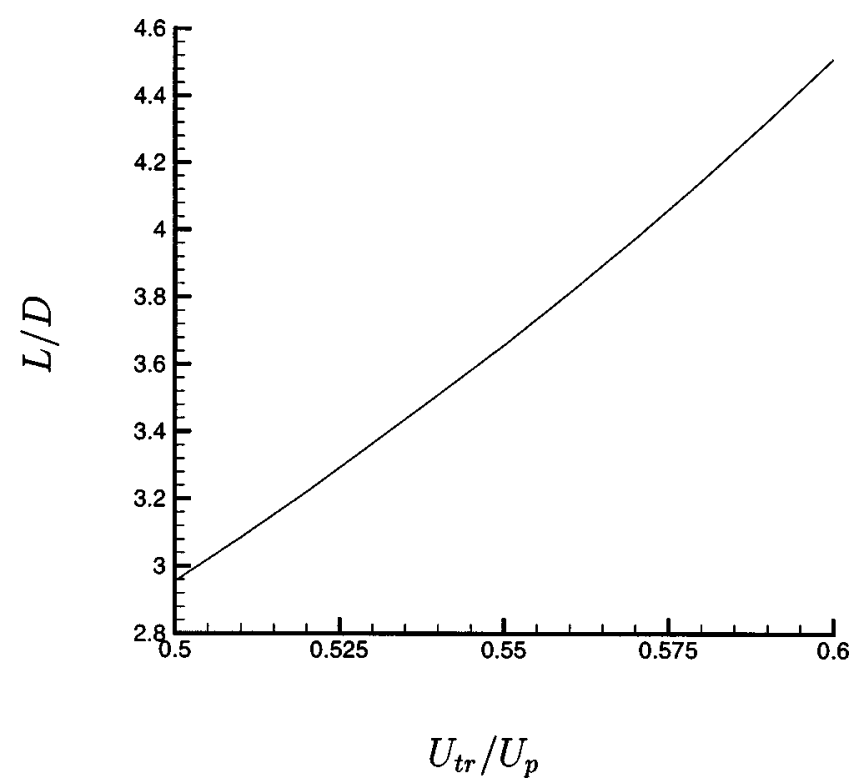

FIG. 2. Variation of formation number $(L / D)$ with nondimensional translation velocity $\left(U_{\mathrm{tr}} / U_{p}\right)$.

limitations of the cylinder-piston mechanism in producing a very thick vortex ring similar to Hill's vortex. In fact, careful examination of Figs. 1 and 2 suggests that to produce a Hilllike vortex one needs a vortex generator that can deliver more energy compared to the simple cylinder-piston mechanism with the same circulation and impulse. One design could be based on a cylinder with a time varying exit diameter.

The fact that the pinch-off parameters are independent of the piston velocity tells us that the universal time scale of vortex formation does not depend strongly on the piston velocity profile. This is experimentally confirmed in Ref. 2. On the other hand, it is expected that the velocity profile at the cylinder outlet has a profound effect on the final stage and on the formation number. This can be manifested as large variations of the invariants of motion to variations in the velocity profile at the cylinder outlet. This variation of formation time scale with exit velocity profile has also been observed in experiments by Gharib et al. ${ }^{2}$

As predicted by Benjamin ${ }^{8}$ and experimentally observed by Gharib et al. ${ }^{2}$ there is a limiting value of $E_{\text {nd }}$ around 0.33 . The value predicted for $E_{\text {nd }}$ in our model is $0.4 \gtrsim E_{\text {nd }} \gtrsim 0.27$ for $U_{\text {tr }}$ varying between $0.5 U_{p}$ and $0.6 U_{p}$ (see Fig. 1), which is in good agreement with the experimental result. Furthermore Gharib et $a .^{2}$ showed that for each experimental setup there is a maximum achievable circulation for the leading vortex for various $L / D$. Here we suggest that if we nondimensionalize the circulation as suggested by Eq. (17), all of the circulation curves in Ref. 2 fall into a single curve that shows a maximum achievable circulation for the leading vortex. Using our analytical model we predict that this limiting nondimensional circulation $\Gamma_{\text {nd }}$ (calculated based on $U_{\text {tr }}$ ) falls in the range $1.77 \leq \Gamma_{\mathrm{nd}} \leq 2.07$, which indicates that steady vortex rings with higher $\Gamma_{\text {nd }}$ is not possible. We have calculated this quantity for the leading pinched off vortex in the set of experiments by Gharib et al. ${ }^{2}$ and found that it is indeed a constant, namely $\Gamma_{\mathrm{nd}} \approx 1.75 \pm 0.05$.

Finally we would like to note that the parameters involved in our dimensional analysis, $E, \Gamma, I$, and $t U_{\mathrm{tr}} / D$ are the characteristics of the resulting vortex ring and not of the generation process. Other mechanisms for vortex generation can be analyzed with our method if the first approximations for the invariants of motion $E, \Gamma, I$ are known. An example of this is the numerical generation of a vortex ring by a nonconservative force.

\section{ACKNOWLEDGMENTS}

We would like to thank K. Shariff of NASA Ames Research Center for his interest in this work. M. G. would like to acknowledge the support by ONR Grant No. URI N00014-91-J-1610.

${ }^{1}$ K. Mohseni, "Statistical equilibrium theory for axi-symmetric flows", (to be published).

${ }^{2}$ M. Gharib, E. Rambod, and K. Shariff, "A universal time scale for vortex ring formation,' J. Fluid Mech. 360, 121 (1998).

${ }^{3}$ J. Norbury, "A family of steady vortex rings," J. Fluid Mech. 57, 417 (1973).

${ }^{4}$ J. P. Sullivan, S. E. Widnall, and S. Ezekiel, "Study of vortex rings using a laser doppler velocimeter," AIAA J. 11, 1384 (1973).

${ }^{5}$ Y. H. Wan, "Variational principles for Hill's spherical vortex and nearly spherical vortices," Trans. Am. Math. Soc. 308, 299 (1988).

${ }^{6}$ P. H. Roberts, "A Hamiltonian theory for weakly interacting vortices," Mathematika 19, 169 (1972).

${ }^{7}$ A. Friedman and B. Turkington, "Vortex rings: Existence and asymptotic estimates," Trans. Am. Math. Soc. 268, 1 (1981).

${ }^{8}$ T. B. Benjamin, "The alliance of practical and analytical insights into the nonlinear problems of fluid mechanics," in Applications of Methods of Functional Analysis to Problems in Mechanics (Springer-Verlag, Berlin, 1976), Vol. 503, pp. 8-29. 